\title{
Using a Nonlinear Disturbance Observer to Estimated the Human Force Applied to a Two-wheeled Cane For Walking Assistance
}

\author{
Phi Van Lam \\ Dept. Electrical and Computer Engineering \\ Yokohama National University \\ Kanagawa, Japan \\ www.pvl.vn@gmail.com \\ or phi-lam-pv@ynu.jp
}

\author{
Tomoyuki Shimono, Yasutaka Fujimoto \\ Dept. Electrical and Computer Engineering \\ Yokohama National University \\ Kanagawa Institute of Industrial Science and Technology \\ Kanagawa, Japan \\ shimono-tomoyuki-hc@ynu.ac.jp \\ fujimoto@ynu.ac.jp
}

\begin{abstract}
Applying a nonlinear disturbance observer to a twowheeled cane for walking assistance is proposed in this paper. The hardware design and controller for the two-wheeled cane are also presented. The two-wheeled cane is designed to help users, especially the elderly walk and balance. The design of the two-wheeled cane is based on an inverted pendulum model, and to validate the performance of system multiple simulation and experimental tests were performed with the designed hardware model. The nonlinear disturbance observer presented good results, which were the same as the force sensor measurement signals. The experimental results show that the two-wheeled cane based on the nonlinear disturbance observer is useful to help elderly persons maintain their balance.
\end{abstract}

Index Terms-Inverted pendulum, nonlinear disturbance observer, two-wheeled cane, robotic cane, assist devices.

\section{INTRODUCTION}

The population of the aging community is expanding rapidly, especially in developed countries, as are the number of services designed to support elderly people with compact and flexible devices; this is the main target of all researchers.

For example, Refs. [1], [2] tried to reduce the size of a traditional cane using an omnidirectional wheel to help users maintain their balance; however, such a system would be dangerous for elderly people if the speed of the robotic cane is fast and in an uncontrolled state. However, to support users in a rehabilitation center, Ref. [3] studied movement using a three-wheeled robot; this designed was less flexible, and its application in practice was limited because it was hard to use when walking on rugged terrain.

There are several compact assistant devices such as robotic canes to help users maintain their balance; however, most results only detail the robotic system [4] or simulation results

[5], [6] without providing experimental results to prove the true utility of the proposed controller.

In Ref. [7], experimental results of a two-wheeled cane model were presented. However, the mechanical structure was quite complex and used an inverted pendulum model with

We would like to thank Otsuka Toshimi Scholarship Foundation for funding for the doctoral scholarship. a type of grip handle that makes it difficult to walk and a force feedback from the robot to the user was not proposed; therefore, a user would have a liter support from such a type of two-wheeled cane. Moreover, a basic control stable of an inverted pendulum model base on dynamic surface control with nonlinear disturbance observer was presented in Ref. [8] without any force estimation applied to the rod of the system.

To overcome the drawbacks in the above studies, we propose a two-wheeled cane based on an inverted pendulum model. Parallel to the design of the two-wheeled cane and the controller is a test with actual users. In addition, the performance of the nonlinear disturbance observer when estimating the human force applied to the two-wheeled cane was compared to a force sensor, which obtained the same results.

This paper contains five sections. In Section II, we present the hardware of the two-wheeled cane in detail. In Section III, we solve the mathematical equations for the two-wheeled cane, including the Lie algebra method, and the nonlinear disturbance observer is developed to evaluate the parameters of the controller. In Section IV, the performance of the nonlinear disturbance observer applied to the controller is discussed via experimental results. Finally, the conclusions and future work are described in Section V.

\section{HARDWARE OF THE TWO-WHEELED CANE BASED ON INVERTED PENDULUM MODELING}

In this section, the hardware of the two-wheeled cane is presented.

A two-wheeled cane is designed as shown in Fig. 1. It has a handle on the top of the cane connected to the frame of the two-wheeled cane via a rod. This frame was printed using a computer numerical control machine our laboratory and is connected to two natural rubber tire electric wheels (Fig. 2) on the left and right sides. There are two motor drivers on the left and right sides of the frame to control the output current of $10 \mathrm{~A}$ to the brushless motor. At the center of the frame, there are Li-ion batteries with a capacity of $36 \mathrm{~V} / 4400 \mathrm{mAh}$ to ensure that the two-wheeled cane can operate for more than 


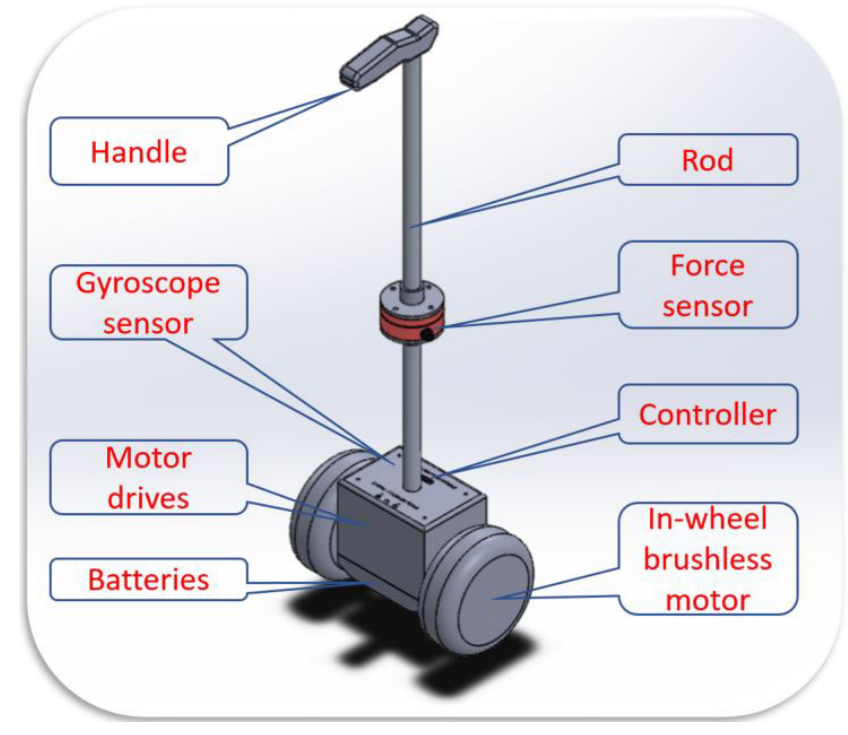

Fig. 1. Hardware design of a two-wheeled cane for walking assistance.

$10 \mathrm{~h}$ without charging. On the bottom, there are a Raspberry Pi Zero W controller and a bridge circuit connected to an accelerometer and a gyroscope sensor to calculate and control the motion of the two-wheeled cane based on our algorithm.

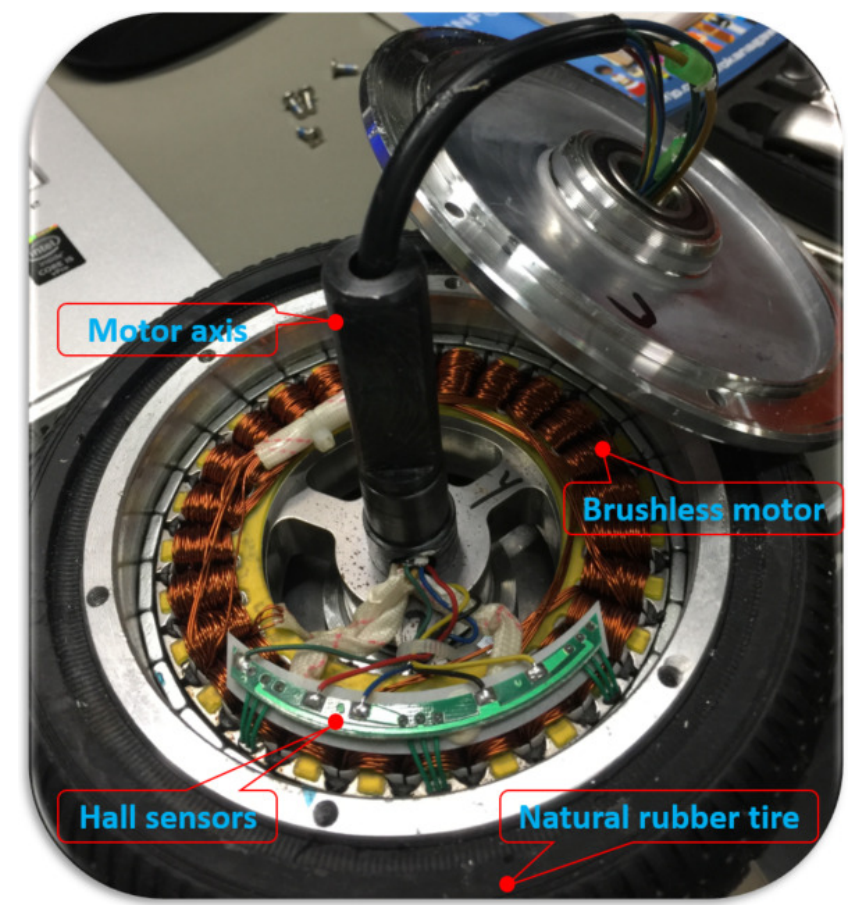

Fig. 2. Natural rubber tire electric wheel including a brushless motor and Hall sensors.

The natural rubber tire electric wheel includes a brushless motor and Hall sensors, as shown in Fig. 2. Using this structure, the two-wheeled cane can work well in any environment more details concerning the structure are given in Table I. Due to the special structure of the wheel, which does not include any gearbox types, the two-wheeled cane can operate silently and can rotate with a small power connection, which is not possible with other motor-included gear types.

TABLE I

PARAMETERS OF THE NATURAL RUBBER TIRE ELECTRIC WHEELS

\begin{tabular}{|l|c|c|c|}
\hline Explanation & Symbol & Unit & Values \\
\hline Voltage & $\mathrm{V}$ & $\mathrm{V}$ & 36 \\
\hline Output power & $\mathrm{P}$ & $\mathrm{W}$ & 250 \\
\hline Hall sensor & $\mathrm{H}$ & $\mathrm{PPR}$ & 90 \\
\hline Size (thickness x diameter nominal) & $\mathrm{S}$ & $\mathrm{mm}$ & $46 \times 168$ \\
\hline
\end{tabular}

In addition, the MPU6050 accelerometer and gyroscope sensor (Fig. 3) are low-price items. The MPU6050 devices are connected to a 3-axis gyroscope, with a selectable range up to $\pm 2000 \mathrm{degree} / \mathrm{s}$, and a 3-axis accelerometer, with a selectable range up to $\pm 8 \mathrm{~g}$ and $400 \mathrm{kHz}$, with a fast mode $I^{2} C$ (InterIntegrated Circuit) to communicate with all registers to easily connect to the Raspberry Pi Zero W controller.

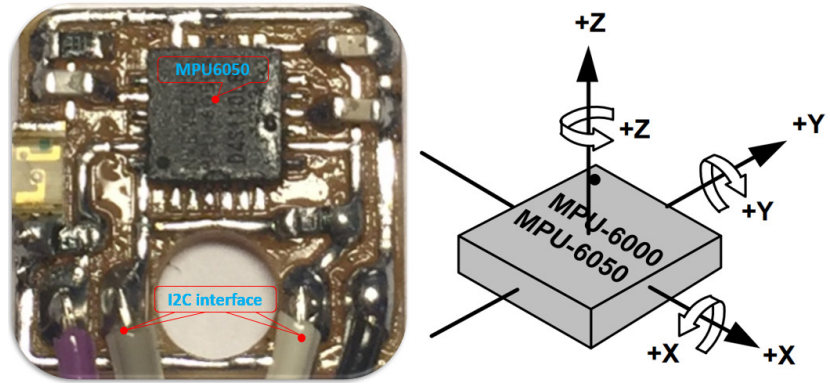

Fig. 3. Designed circuit and orientation of axes of sensitivity and polarity of rotation of gyroscope sensor - MPU6050.

\section{MATHEMATICAL MODEL BASED ON AN INVERTED PENDULUM MODEL AND THE DESIGN OF THE TWO-WHEELED CANE CONTROLLER SYSTEM}

A. Mathematical model of a two-wheeled cane using an inverted pendulum model

The two-wheeled cane in Fig. 4 is based on an inverted pendulum model.

TABLE II

EXPLANATION OF SYMBOLS

\begin{tabular}{|l|c|c|}
\hline Explanation & Symbol & Unit \\
\hline Inertia of rod & $J_{\phi}$ & $\mathrm{kg} \cdot \mathrm{m}^{2}$ \\
\hline Inertia of wheel & $J_{\theta}$ & $\mathrm{kg} \cdot \mathrm{m}^{2}$ \\
\hline Viscous friction coefficient of rod & $D_{\phi}$ & $\mathrm{N} . \mathrm{m} . \mathrm{s} / \mathrm{rad}$ \\
\hline Viscous friction coefficient of wheel & $D_{\theta}$ & $\mathrm{N} . \mathrm{m} . \mathrm{s} / \mathrm{rad}$ \\
\hline Actuation torque & $\tau$ & $\mathrm{N} . \mathrm{m}$ \\
\hline Gravitational acceleration & $g$ & $\mathrm{~m} / \mathrm{s}^{2}$ \\
\hline Angle of the rod & $\phi$ & $\mathrm{rad}$ \\
\hline Angle of the wheel & $\theta$ & $\mathrm{rad}$ \\
\hline Disturbance according to $\phi$ & $d_{1}$ & $\mathrm{~N} . \mathrm{m}$ \\
\hline Disturbance according to $\theta$ & $d_{2}$ & $\mathrm{~N} . \mathrm{m}$ \\
\hline
\end{tabular}




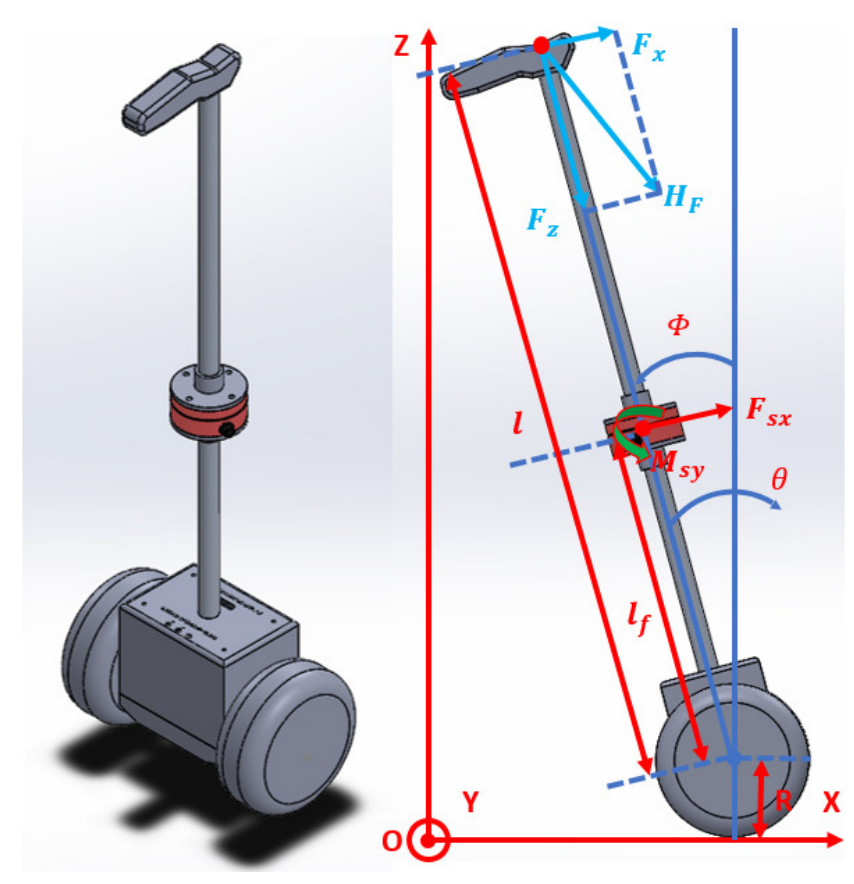

Fig. 4. Coordinate system of two-wheeled cane using an inverted-pendulum model.

The rod has a length of $l$, the wheel has a radius of $r$, the mass of the wheels is $M$, and mass of the rod is $m$ and more details as shown in Table II. Analyzing this system, we use the Lagrange equation to determine the motion of an inverted pendulum as follows:

$$
\begin{aligned}
& \frac{d}{d t}\left(\frac{\partial L}{\partial \dot{\phi}}\right)-\frac{\partial L}{\partial \phi}=0-d_{1} \\
& \frac{d}{d t}\left(\frac{\partial L}{\partial \dot{\theta}}\right)-\frac{\partial L}{\partial \theta}=\tau-d_{2}
\end{aligned}
$$

An analysis of an inverted pendulum model was presented in Ref. [1], [2]. Similarly, the motion equation of the system is as follows:

$$
\left[\begin{array}{ll}
H_{11} & H_{12} \\
H_{21} & H_{22}
\end{array}\right]\left[\begin{array}{l}
\ddot{\phi} \\
\ddot{\theta}
\end{array}\right]+\left[\begin{array}{l}
b_{1} \\
b_{2}
\end{array}\right]=\left[\begin{array}{c}
-d_{1} \\
\tau-d_{2}
\end{array}\right]
$$

where,

$$
\begin{aligned}
H_{11} & =J_{\theta}+(M+m) r^{2}+2 m r l \cos \phi+J_{\phi}+m l^{2} \\
H_{12} & =H_{21}=-J_{\theta}-(M+m) r^{2}-m r l \cos \phi \\
H_{22} & =J_{\theta}+(M+m) r^{2} \\
b_{1} & =-\dot{\phi}^{2} m r l \sin \phi-m g l \sin \phi+D_{\phi} \dot{\phi} \\
b_{2} & =\dot{\phi}^{2} m r l \sin \phi+D_{\theta} \dot{\theta}
\end{aligned}
$$

A two-wheeled cane requires a torque to be applied to the axis motor to move and help users maintain balance. The torque is derived from (3) as shown below:

$$
\tau=\left(H_{22}-\frac{H_{12} H_{21}}{H_{11}}\right) u-\frac{H_{21} b_{1}}{H_{11}}+b_{2}-d_{2}
$$

B. Linearization of the nonlinear system using the Lie algebra method

We expand the motion equation of the two-wheeled cane in (10). We can easily recognize that this system is a nonlinear system.

$$
\dot{x}=\left[\begin{array}{c}
\dot{\phi} \\
\ddot{\phi} \\
\dot{\theta} \\
\ddot{\theta}
\end{array}\right]=\left[\begin{array}{c}
\dot{\phi} \\
-\frac{b_{1}}{H_{11}} \\
\theta \\
0
\end{array}\right]+\left[\begin{array}{c}
0 \\
-\frac{H_{12}}{H_{11}} \\
0 \\
1
\end{array}\right] u
$$

The Lie algebra method to linearize a nonlinear system is one of the best methods to control the inverted pendulum model. This method is defined by the functions given in (11) and (12):

$$
\begin{aligned}
& \dot{x}=f(x)+g(x) u \\
& y=h(x)
\end{aligned}
$$

After expanding these equations using the Lie algebra method to find the $y$ function as a linear equation, its derivatives from first to third order can be calculated using (13)-(16).

$$
\begin{aligned}
y & =\int_{0}^{\phi} \frac{H_{11}}{H_{12}} d \phi+\theta \\
\dot{y} & =\frac{H_{11}}{H_{12}} \dot{\phi}+\dot{\theta} \\
\ddot{y} & =\frac{\partial}{\partial \phi} \frac{H_{11}}{H_{12}} \dot{\phi}^{2}-\frac{b_{1}}{H_{12}} \\
y^{(3)} & \simeq \frac{\partial^{2}}{\partial \phi^{2}} \frac{H_{11}}{H_{12}} \dot{\phi}^{3}-\frac{\partial}{\partial \phi} \frac{b_{1}}{H_{12}} \dot{\phi}-2\left(\frac{\partial}{\partial \phi} \frac{H_{11}}{H_{12}}\right) \frac{b_{1}}{H_{11}} \dot{\phi}
\end{aligned}
$$

Obviously, these equations are the linear equations of the nonlinear system that show the motion of the two-wheeled cane.

Higher derivatives results is a smoother system. However, depending on the controller, there may not be sufficient speed to calculate the motion equations of the system. In this case, we use the fourth-order derivative, which meets the speed required to process a basic feedback loop controller to control this system; the input value is given by (17).

$$
u=\frac{v-L_{f}^{4} h(x)}{L_{g} L_{f}^{3} h(x)}
$$

where,

$$
\begin{aligned}
L_{g} L_{f}^{3} h(x)= & -3 \frac{\partial^{2}}{\partial \phi^{2}} \frac{H_{11}}{H_{12}} \frac{H_{12}}{H_{11}} \dot{\phi}^{2}+\frac{\partial}{\partial \phi} \frac{b_{1}}{H_{12}} \frac{H_{12}}{H_{11}} \\
& +2 \frac{\partial}{\partial \phi} \frac{H_{11}}{H_{12}} \frac{H_{12}}{H_{11}{ }^{2}} b_{1} \\
L_{f}{ }^{4} h(x)= & -\frac{\partial^{2}}{\partial \phi^{2}} \frac{b_{1}}{H_{12}} \dot{\phi}^{2}+\frac{\partial^{3}}{\partial \phi^{3}} \frac{H_{11}}{H_{12}} \dot{\phi}^{4} \\
& -5\left(\frac{\partial^{2}}{\partial \phi^{2}} \frac{H_{11}}{H_{12}}\right) \frac{b_{1}}{H_{11}} \dot{\phi}^{2}-2 \frac{\partial}{\partial \phi} \frac{b_{1}}{H_{12}} \dot{\phi}^{2} \\
& +\frac{\partial}{\partial \phi} \frac{b_{1}}{H_{12}} \frac{b_{1}}{H_{11}}+2 \frac{\partial}{\partial \phi} \frac{H_{11}}{H_{12}}\left(\frac{b_{1}}{H_{11}}\right)^{2}
\end{aligned}
$$


C. Nonlinear disturbance observer to estimate the human force applied to the two-wheeled cane

With the external disturbances $d_{1}$ and $d_{2}$ corresponding to $\phi$ and $\theta$, we present the nonlinear disturbance observer via the equations below:

$$
\begin{aligned}
& \dot{\varsigma}=-K \varsigma+K^{2} \frac{\partial L}{\partial \dot{q}}+K\left(\frac{\partial L}{\partial q}+\tau_{a l l}\right) \\
& \hat{d}=\varsigma-K \frac{\partial L}{\partial \dot{q}}
\end{aligned}
$$

where, $\frac{\partial L}{\partial \dot{q}}$ has two cases correspond to $\phi$ and $\theta$ as below:

$$
\begin{aligned}
& \frac{\partial L}{\partial \dot{\phi}}=H_{11} \dot{\phi}+H_{12} \dot{\theta} \\
& \frac{\partial L}{\partial \dot{\theta}}=H_{21} \dot{\phi}+H_{22} \dot{\theta}
\end{aligned}
$$

As shown in Fig. 4, the human force $H_{F}$ applied to the rod of the two-wheeled robot has two directions: along the $x$-axis and along the $z$-axis. This estimated force $\hat{d}_{1}$ and $\hat{d}_{2}$ can be calculate by:

$$
\left[\begin{array}{l}
\hat{d}_{1} \\
\hat{d}_{2}
\end{array}\right]=\left[\begin{array}{cc}
-l \cos \phi-r & -l \sin \phi \\
r & 0
\end{array}\right]\left[\begin{array}{l}
\hat{f}_{x} \\
\hat{f}_{z}
\end{array}\right]
$$

From (23), we can estimate the human force on the $z$-axis:

$$
\hat{f}_{z}=-\frac{1}{r l \sin \phi}\left(r \hat{d}_{1}+(l \cos \phi+r) \hat{d}_{2}\right)
$$

Also, the presenter [5], we has $\theta$ and $\dot{\theta}$ as below:

$$
\begin{gathered}
\theta_{\text {ref }}=\theta_{\text {current }} \\
\dot{\theta}_{\text {ref }}=K_{I} \int \hat{d}_{2} d t
\end{gathered}
$$

In this way, we can use this human force make the plan to control the motion of the two-wheeled cane to support the users in maintaining their balance.

\section{EXPERIMENTAL RESULTS}

\section{A. Stabilization of a two-wheeled cane by itself}

The two-wheeled cane is designed to be able to balance with or without being held by a user. This is the basic working mode of an inverted pendulum. Experiments of the controller on the two-wheeled cane have been recorded in videos, and several frames are cut from a video and shown in Fig. 5.

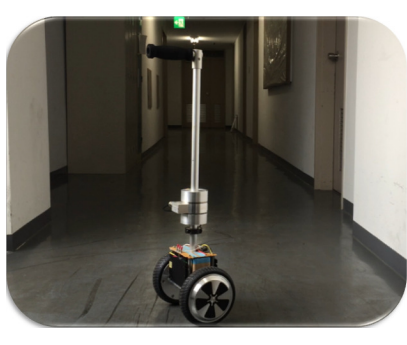

a) $\mathrm{t}=10.0 \mathrm{~s}$

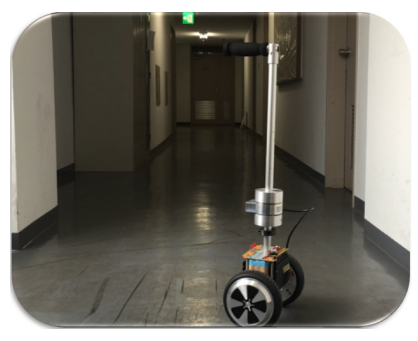

b) $\mathrm{t}=900.0 \mathrm{~s}$
Fig. 5. Two-wheeled cane stability by itself.
The angle of the two-wheeled cane is related to the angle of the cane, which is determined by the gyroscope sensor in Fig. 6; the result shows that the two-wheeled cane achieves a stability point after only $0.05 \mathrm{~s}$ with a large tilt angle of the rod of approximately $0.00008786 \mathrm{rad}$ at $0.02 \mathrm{~s}$ and remains unchanged at $0 \mathrm{rad}$ in the last period.

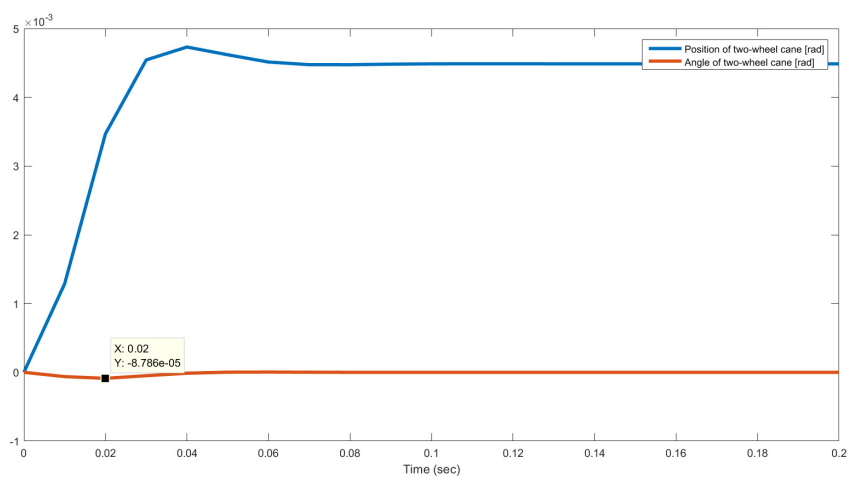

Fig. 6. Angle of the two-wheeled cane when stabilizing itself.

This result shows that using the Lie algebra method, we can linearize the nonlinear system not only around the zero point, as in the linear-quadratic regulator method, but also around large tilt angles of the rod. Therefore, this method is very good at controlling the two-wheeled cane to help users maintain their balance when the tilt angle is equal to or greater than zero degrees.

\section{B. Nonlinear disturbance observer estimations}

As shown in Fig. 8, the CFS080CS102A torque sensor (Tab. III) is mounted on the rod of the two-wheeled cane to determine the actual torque applied to the robot.

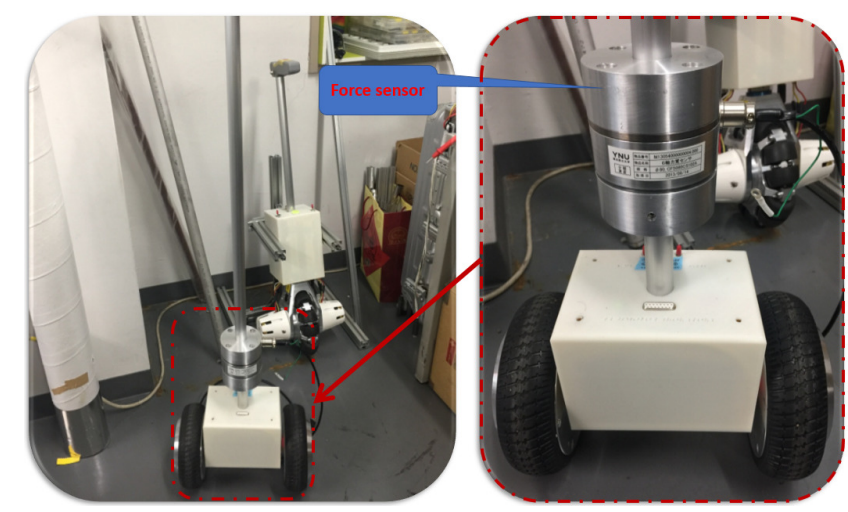

Fig. 7. Torque sensor mounted on the rod to measure the human force applied to the two-wheeled cane.

The actual torque $d$ applied to the motor axis of the twowheeled cane is calculated in Fig. 4 via:

$$
d=F_{s x} l_{f}+M_{s y}
$$

where, $F_{s x}$ is the force along the $x$-axis; $M_{s y}$ is the moment on the $y$-axis, and $l_{f}$ is the distance from the center of the 
TABLE III

PARAMETERS OF THE CFS080CS102A 6 AXIS TORQUE SENSOR.

\begin{tabular}{|c|c|c|}
\hline Explanation & Value & Unit \\
\hline Power supply voltage & 5 & $\mathrm{~V}$ \\
\hline Rated load Fx,Fy,Fz & 1000 & $\mathrm{~N}$ \\
\hline Rated load Mx,My,Mz & 30 & $\mathrm{~N} . \mathrm{m}$ \\
\hline Interface RS422 & 460800 & $\mathrm{bps}$ \\
\hline
\end{tabular}

motor axis to the center of the torque sensor, as shown in Fig. 4.

In Fig. 8, our nonlinear disturbance observer used to estimate the human force applied to the two-wheeled cane obtains the same result as the torque sensor mounted on the rod in all periods of the three cases: standing, fall forward and fall backward.

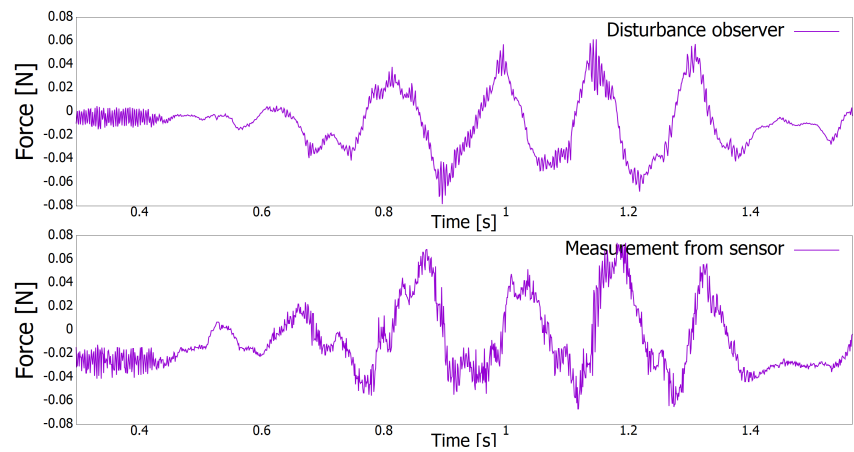

Fig. 8. Human torque estimated by the nonlinear disturbance observer and torque measured by the torque sensor.

The human force applied to the rod of the two-wheeled cane will fluctuate around the zero points when supporting users when standing, as shown in the first period from $0 \mathrm{~s}$ to $0.7 \mathrm{~s}$.

In the next step, when a user starts falling forward from $0.7 \mathrm{~s}$ to $0.8 \mathrm{~s}$, the human force applied to the rod of the twowheeled cane is, for example, a positive $0.06 \mathrm{~N}$ in the head direction of the two-wheeled cane.

Similarly, in the case when a user starts falling backward, the human force measured by torque sensor has negative value, e.g..0.05N, similar to our disturbance observer estimation from $0.8 \mathrm{~s}$ to $0.9 \mathrm{~s}$.

More details of our estimations of these three user cases are repeated in other periods in Fig. 8.

\section{To support users in maintaining their balance}

In the second test, the efficiency of the two-wheeled cane is shown by the angle and feedback position of the two-wheeled cane in Fig. 9.

We present three cases as shown in (Fig. 10):

- Case one is when users need to remain stable from $0 \mathrm{rad}$ to $2.2 \mathrm{rad}$ in the first period in the upright standing position; in case, the two-wheeled cane helps users achieve a balancing point through the handle with the angle and the position of the two-wheeled cane vibration around equilibrium point of $0.01 \mathrm{rad}$ and $0.04 \mathrm{~m}$ respectively;
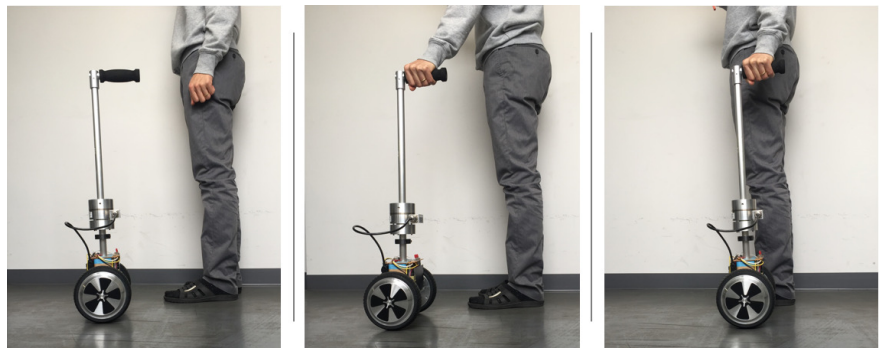

Fig. 9. The two-wheeled cane help users to stand.

- Case two is when the cane supports the movement of the user. In this case, the position of the two-wheeled cane is based on the angle of the rod compared to the stability point and the estimated torque applied to the rod, which establishes a suitable position to help users maintain their balance while they are moving from $2.2 \mathrm{~s}$ to $2.6 \mathrm{~s}$;

- Case three as shown in the last period shown that, when the users tend to fall backward or fall forward, the figure indicates that when the angle of the two-wheeled cane being change to plus or minus values, the positions of the cane will be following respectively to helps the users get a new balancing point from 2.6 s to the last period.

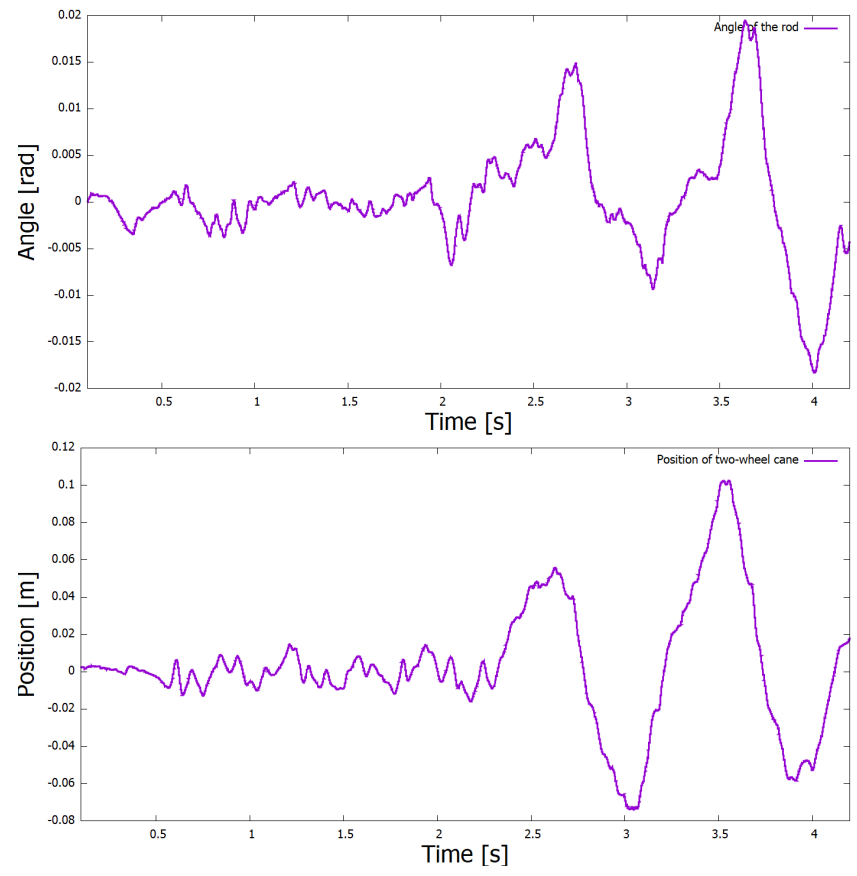

Fig. 10. Angle and position of the two-wheeled cane when it help users maintain their balance.

In the first case, the torque estimation using our disturbance observer is to show in Fig. 11. In the first period from $0 \mathrm{~s}$ to $0.6 \mathrm{~s}$, the user and the two-wheeled cane try to maintain the balancing point together with the human force oscillating between $-0.05 \mathrm{~N}$ and $+0.05 \mathrm{~N}$ before becoming strongly stable in the final period with the human force near the zero point. 


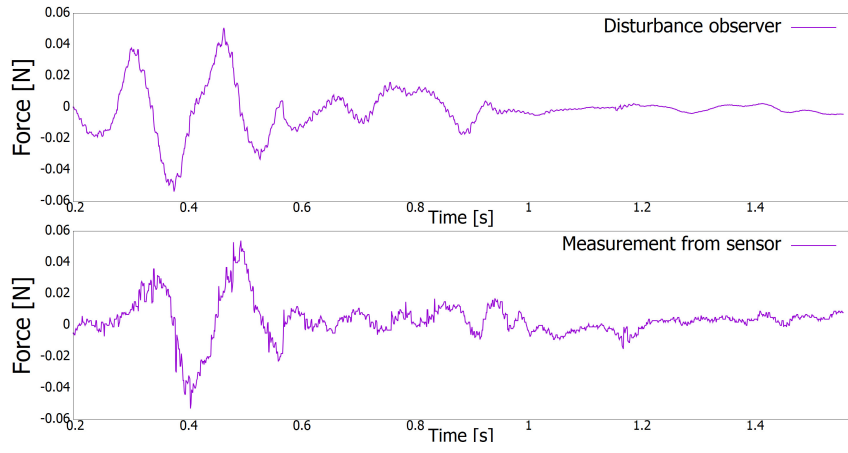

Fig. 11. The two-wheeled cane helping users stand.

In the second case, Fig. 12 shows that, when the user needs to move forward, the cane is tilted at an angle of $\phi$ compared to the vertical axis and the torque estimation. Then, an output torque is applied to the motor axis and this torque is calculated via (9) based on the torque estimation to help the two-wheeled cane change position with a corresponding angle of $\theta$.
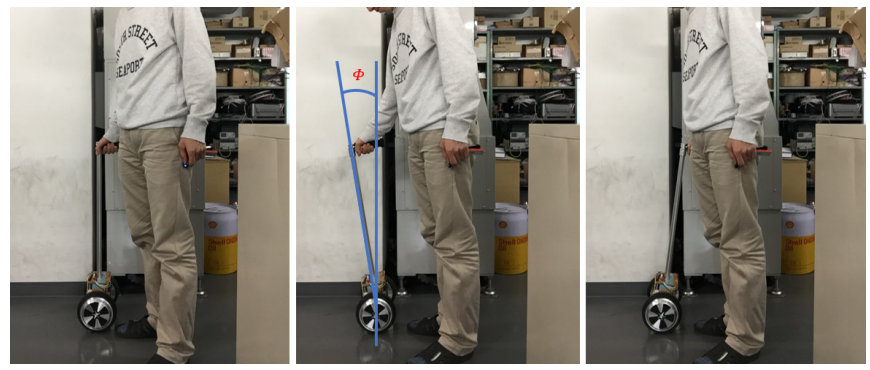

Fig. 12. Three modes of supporting by two-wheeled cane.

In Fig. 13, the two-wheeled cane helps users stand; however, in some cases, the users started to fall backward and then the robot detects the human force applied to the rod of the twowheeled cane to change its position to support the user in finding a new standing position (in the last period of $2.6 \mathrm{~s}$ to 3 s Fig. 10).
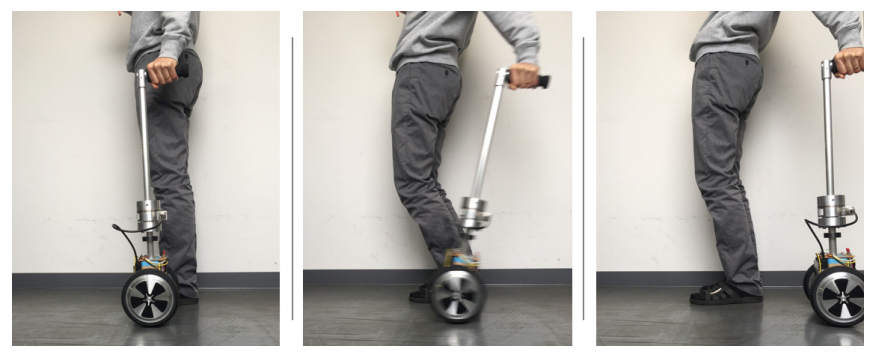

Fig. 13. The two-wheeled cane helping a users balance when they are starting to fall backward.

Similarly, in the last period of $3 \mathrm{~s}$ to $3.5 \mathrm{~s}$ in Fig. 10 when a user starts to fall forward, the two-wheeled cane can help them stand or maintain their balance around the equilibrium point (Fig. 14).

In this way, with the estimated human force applied to the rod of the two-wheeled cane, the controller controls the
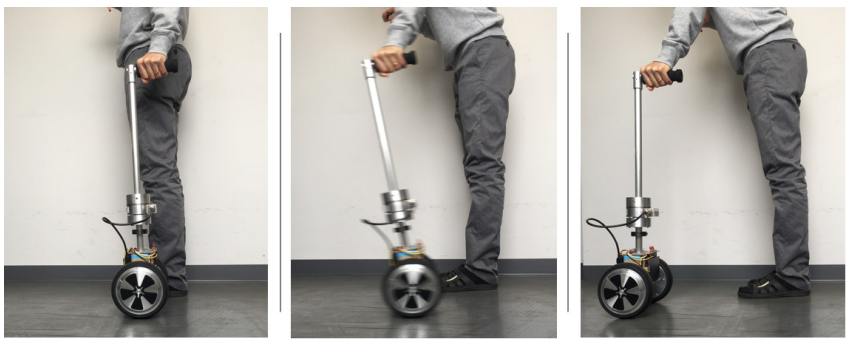

Fig. 14. The two-wheeled cane helping a users balance when they are starting to fall forwards.

position and speed of the robot to help the user maintain their balance when they walk or start to fall backward or forward.

\section{CONCLUSIONS AND FUTURE WORK}

We designed the hardware of the two-wheeled cane based on a natural rubber tire electric wheel with a high-speed processing controller for walking assistance.

The experimental results on the hardware indicate that the two-wheeled cane is effective in helping users maintain their balance and our nonlinear disturbance observer provides a good method to estimate the human force applied to the twowheeled cane for walking assistance.

In future work, we intend to reduce the size of the system while increasing the capacity of the battery to increase the running time of the two-wheeled cane without charging. Moreover, we will further test the two-wheeled cane at the rehabilitation center to improve it.

\section{ACKNOWLEDGMENTS}

We would like to thank Otsuka Toshimi Scholarship Foundation for funding for the doctoral scholarship.

\section{REFERENCES}

[1] P. V. Lam and Y. Fujimoto, "Building and test a controller of the robotic cane for walking assistance," The IEEJ International Workshop on Sensing, Actuation, Motion Control, and Optimization, vol. 3, pp. SS2-6, Mar. 2017.

[2] P. V. Lam and Y. Fujimoto, "Completed hardware design and controller of the robotic cane using the inverted pendulum for walking assistance," 2017 IEEE 26th International Symposium on Industrial Electronics (ISIE), vol. 19-21 June 2017, pp. 2163-5145, Aug. 2017.

[3] S. Nakagawa, Y. Hasegawa, T. Fukuda, I. Kondo, M. Tanimoto, P. Di, J. Huang, and Q. Huang, "Tandem stance avoidance using adaptive and asymmetric admittance control for fall prevention," IEEE Transactions on Neural Systems and Rehabilitation Engineering, vol. 24, DOI 10.1109/TNSRE.2015.2429315, no. 5, pp. 542-550, May. 2016.

[4] Y. Ota, M. Ryumae, and S. Keiichi Sato, "Robotic cane devices," United States Patent Application Publication, no. US 2013/0041507 A1, 2013.

[5] K. Shimizu and Y. Fujimoto, "A robotic cane for walking support using two control modes," IEEJ International Workshop on Sensing, Actuation, Motion Control, and Optimization, pp. TT9-5, Mar. 2016.

[6] K. Shimizu, S. A. Issam, and Y. Fujimoto, "A robotic cane for walking assistance,", IEEJ International Power Electronics Conference (IPEC), DOI 10.1109/IPEC.2014.6869857, pp. 1968-1973, May. 2014.

[7] P. V. Lam and Y. Fujimoto, "Two-wheel cane for walking assistance," IEEJ Int. Power Electronics Conference (IPEC), May. 2018.

[8] J. Huang, S. Ri, L. Liu, Y. Wang, J. Kim, and G. Pak, "Nonlinear disturbance observer based dynamic surface control of mobile wheeled inverted pendulum," IEEE Transactions on Control Systems Technology, vol. 23, DOI 10.1109/TCST.2015.2404897, no. 6, pp. 2400-2407, Nov. 2015 